\title{
Metodología para identificar las estrategias de mercadeo que afectan en el momento de compra: TRAPPOLA
}

Methodology to identify the marketing strategies that affect the purchasing moment: TRAPPOLA

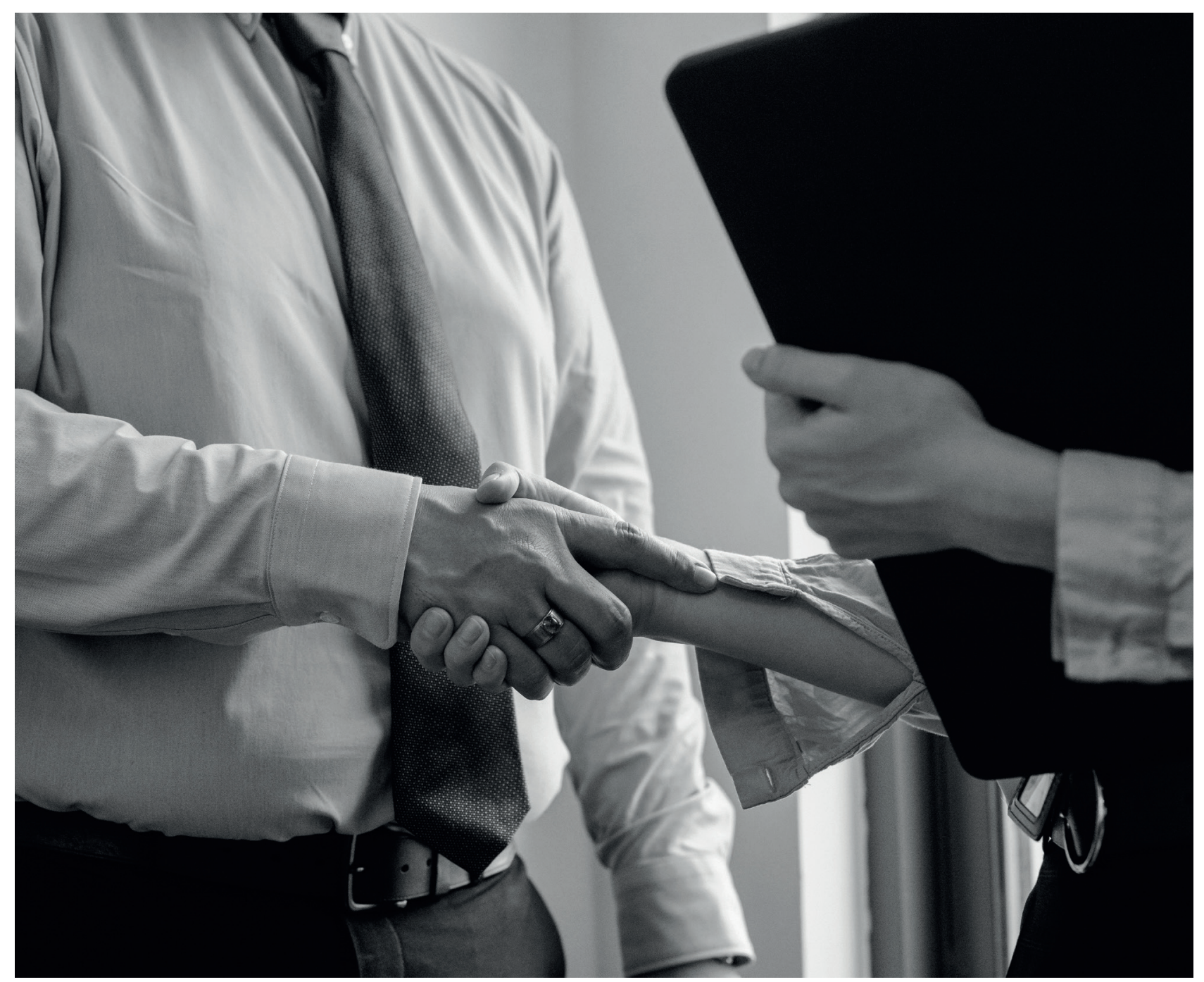

Fuente: Pexels.com Licencia Creative Commons 


\title{
Metodología para identificar las estrategias de mercadeo que afectan en el momento de compra: TRAPPOLA ${ }^{1}$
}

\author{
Methodology to identify the marketing strategies that affect the \\ purchasing moment: TRAPPOLA
}

\section{Angie Paola Agudelo Loaiza², Catalina Londoño Velásquez³, Ginette Peláez Rodríguez ${ }^{4}$, Juan Pablo Méndez Ríos ${ }^{5}$, Julián Andrés Gutiérrez Castaño ${ }^{6}$}

\author{
Artículo recibido en abril 28 de 2020; artículo aceptado en julio 22 de 2020
}

\begin{abstract}
Este artículo puede compartirse bajo la Licencia Creative Commons Atribución-NoComercial-Compartirlgual 4.0 Internacional y se referencia usando el siguiente formato: Agudelo, A. P., Londoño, C., Peláez, G. Méndez, J. P, y Gutiérrez, J. A. (2021). Metodología para identificar las estrategias de mercadeo que afectan en el momento de compra: TRAPPOLA. I+D Revista de Investigaciones, 16(1), 45-52. http://dx.doi.org/10.33304/revinv.v16n1-2021004.
\end{abstract}

\begin{abstract}
Resumen
La creciente globalización, el consumismo y la rivalidad entre las empresas han influido a lo largo del tiempo en la generación de estrategias de mercadeo para prevalecer, por medio del análisis de los comportamientos humanos. Por ello, a partir de una revisión de literatura de estos aspectos, se diseñó una metodología constructivista llamada "Trappola", que permite, a través de una situación de consumo enfocada en estrategias de mercadeo, que los participantes vivencien el principio de la economía conductual, que se basa en la idea de que las personas no son completamente racionales, sino también previsiblemente irracionales. Es decir, que su irracionalidad se produce siempre del mismo modo una y otra vez. Por ende, el objetivo de aprendizaje gira en torno a reconocer métodos como el efecto señuelo, efecto anclaje, coherencia arbitraria, gregarismo o efecto arrastre, efecto del coste cero, marketing emocional y efecto de la expectativa o efecto Pigmalión. Por último, se concluye que el comportamiento humano al ser predecible permite que las estrategias de marketing se cumplan en gran medida al momento de efectuar una compra.
\end{abstract}

Palabras clave: Aprendizaje activo, economía de mercado, educación alternativa, mercadeo.

\footnotetext{
${ }^{1}$ Artículo de investigación, de enfoque cualitativo, artículo no experimental de carácter descriptivo, resultado de un proyecto de investigación en curso, perteneciente al área de ingeniería industrial, subárea de marketing, desarrollado en el grupo de investigación GEIO, financiado por la Universidad Tecnológica de Pereira (Colombia). Dirección: carrera 27 n. ${ }^{\circ}$ 10-02 Barrio Álamos, PBX: +57 63137300 . Fecha de inicio: 25.4.2019.

2 Estudiante de Ingeniería Industrial de la Universidad Tecnológica de Pereira. Vinculado al Grupo de Investigación GEIO, Universidad Tecnológica de Pereira (Colombia). Dirección: carrera 27 n. ${ }^{\circ} 10-02$ Barrio Álamos, PBX: +57 63137300 . ORCIR ID: https://orcid.org/0000-0002-7689-3322. Correo electrónico institucional: angieagudelo17@utp.edu.co.

${ }^{3}$ Estudiante de Ingeniería Industrial de la Universidad Tecnológica de Pereira. Vinculado al Grupo de Investigación GEIO, Universidad Tecnológica de Pereira (Colombia). Dirección: carrera 27 n. 10-02 Barrio Álamos, PBX: +57 6 3137300, ORCIR ID: https://orcid.org/0000-0001-6968-9035. Correo electrónico institucional: catalina.londono1@utp.edu.co.

${ }^{4}$ Estudiante de Ingeniería Industrial de la Universidad Tecnológica de Pereira. Vinculado al Grupo de Investigación GEIO, Universidad Tecnológica de Pereira (Colombia). Dirección: carrera 27 n. ${ }^{10}$ 10-02 Barrio Álamos, PBX: +57 6 3137300. ORCIR ID: https://orcid.org/0000-0002-0121-6060. Correo electrónico institucional: g.pelaez@utp.edu.co.

${ }^{5}$ Estudiante de Ingeniería Industrial de la Universidad Tecnológica de Pereira. Vinculado al Grupo de Investigación GEIO, Universidad Tecnológica de Pereira (Colombia). Dirección: carrera 27 n. ${ }^{\circ} 10-02$ Barrio Álamos, PBX: +57 63137300 . ORCIR ID: https://orcid.org/0000-0003-0962-5632. Correo electrónico institucional: pablo.mendez@utp.edu.co.

${ }^{6}$ Estudiante de Ingeniería Industrial de la Universidad Tecnológica de Pereira. Vinculado al Grupo de Investigación GEIO, Universidad Tecnológica de Pereira (Colombia). Dirección: carrera 27 n. ${ }^{\circ}$ 10-02 Barrio Álamos, PBX: +57 6 3137300. ORCIR ID: https://orcid.org/0000-0003-0257-3606. Correo electrónico institucional: julian.gutierrez@utp.edu.co.
} 
Angie Paola Agudelo Loaiza, Catalina Londoño Velásquez, Ginette Peláez Rodríguez, Juan Pablo Méndez Ríos, Julián Andrés Gutiérrez Castaño Metodología para identificar las estrategias de mercadeo que afectan en el momento de compra:TRAPPOLA

\begin{abstract}
The increasing globalization, consumerism, and rivalry between companies have influenced over time the generation of marketing strategies to prevail through the analysis of human behavior. Therefore, based on a literature review of these aspects, a constructivist methodology was designed called "Trappola", which allows participants to experience the principle of behavioral economics through a consumption situation focused on marketing strategies, which is based on the idea that people are not completely rational, but also predictably irrational. That is to say, that their irrationality always occurs in the same way over and over again. Therefore, the learning objective revolves around recognizing methods such as the decoy effect, anchoring effect, arbitrary coherence, gregariousness or dragging effect, zero cost effect, emotional marketing, and the effect of expectation or Pygmalion effect. Finally, it is concluded that human behavior, being predictable, allows marketing strategies to be largely complied with at the moment of making a purchase.
\end{abstract}

Keywords: Active learning, market economy, alternative education, marketing.

\section{Introducción}

Trappola surge a partir de la necesidad de mostrar a las personas las diferentes estrategias de mercadeo utilizadas por las empresas para influir en su comportamiento a la hora de comprar, y así evitar que caigan tan fácilmente en las trampas de mercadeo que no son más que publicidad subliminal o juegos psicológicos que tienen por objetivo generar un mayor consumo. En esta lúdica se emplearán diversas áreas del conocimiento, como mercadeo y administración. Como toda actividad académica, durante su desarrollo se plantea una hipótesis, que se basa en el principio de la economía conductual que postula la idea de que las personas no son completamente racionales, sino también previsiblemente irracionales (Ariely, 2008). Siguiendo este orden de ideas, se podría asumir que el hecho de caer en las trampas del mercado está asociado a la incapacidad humana para ignorar su lado irracional.

En este artículo se propone diseñar una experiencia lúdica que, mediante experimentos, deje en evidencia cómo algunas estrategias de marketing afectan las decisiones de las personas a la hora de comprar, a la vez que se intenta motivar el aprendizaje en técnicas de mercadeo y facilitar la apropiación de los conceptos teóricos mediante la simulación.

Trappola está diseñada de tal forma que los participantes tengan libertad de escoger los productos que ellos "deseen", puesto que no se les dirá que se va a evaluar su forma de comprar, para que su comportamiento sea insesgado, y de esta forma contribuyan a la comprobación o rechazo de la hipótesis.

Trappola estará ambientada en un supermercado donde los participantes, organizados en grupos de tres personas, tendrán dinero didáctico para comprar productos, teniendo como base una lista de necesidades que corresponde a las compras mensuales de su equipo. Dentro del supermercado irán sucediendo una serie de eventos que buscarán modificar el comportamiento de los participantes, quienes estarán dentro del supermercado entre 15 y 25 minutos. Para realizar la compra pasarán a una caja que registrará los productos en el orden de la lista de necesidades, para facilitar un posterior análisis de lo sucedido. Finalmente se realiza una realimentación que permita a los participantes reflexionar sobre los conceptos evidenciados en la lúdica, haciendo énfasis en la presencia de estos en la vida cotidiana.

\section{Marco referencial}

En la actualidad, el comportamiento de los mercados difícilmente sigue el comportamiento usual descrito por la economía tradicional, debido principalmente al cambio en la mente de los consumidores respecto a las campañas de marketing que realizan las compañías. Como lo describe Anderson (2007), las personas buscan cada vez más calidad en los productos que se les ofrecen y capacidad de elección sobre aquellos que realmente vayan acorde con sus gustos; por otra parte, la evolución de la tecnología ha jugado un papel muy importante en la revolución que han llevado a cabo los sujetos que componen el mercado. La aparición de los espacios virtuales como lugares de compra y la posibilidad de los ofertantes de poner a disposición de sus clientes un gran abanico de posibilidades a través de estos medios digitales han llevado a las empresas a tomar diferentes estrategias a la hora de hacer llegar su marca a sus distintos segmentos del mercado.

El marketing es "la actividad, el conjunto de instituciones y los procesos para crear, comunicar, entregar e intercambiar ofertas que tienen valor para los clientes, los socios y la sociedad en general" (American Marketing Association, 2017). En ese orden de ideas, es el mecanismo que ha tenido que desarrollarse a lo largo de los años para que las empresas puedan sobrellevar los cambios del mercado. Así, se logra la denominada dirección del marketing, que es "el arte y la ciencia de seleccionar los mercados meta y lograr conquistar, mantener e incrementar el número de clientes mediante la generación, comunicación y entrega de un mayor valor para el cliente" (Kotler y Keller, 2006). Alrededor de estas definiciones se han creado diferentes formas de realizar marketing, con el fin de cumplir su objetivo de comunicar, 
Angie Paola Agudelo Loaiza, Catalina Londoño Velásquez, Ginette Peláez Rodríguez, Juan Pablo Méndez Ríos, Julián Andrés Gutiérrez Castaño Metodología para identificar las estrategias de mercadeo que afectan en el momento de compra: TRAPPOLA

siempre teniendo en cuenta la forma en que desean recibir información los usuarios; algunos ejemplos son:

- El marketing de sigilo: Es "un acto deliberado de entrar, operar o salir de un mercado de una manera furtiva, secreta o imperceptible, o intentar hacerlo" (Roy y Chattopadhyay, 2010).

- El marketing encubierto: es donde "la identidad del mensaje y/o el remitente del mensaje no están indicados $y$, por lo tanto, el mensaje se transmite al consumidor" (Akdoğan y Altuntas, 2015).

- El marketing extraordinario: Es "el arte de construir cosas [...] en las que vale la pena fijarse. No se trata de aplicar el marketing de cualquier manera, [...] sino de entender que su oferta en sí misma no es extraordinaria, es invisible" (Godin, 2002).

- El marketing sensorial: En el cual, por medio de los sentidos, se busca conectar con las personas y generar una reacción consciente o inconsciente y así influir en su comportamiento (Krishna, 2012).

Los dos primeros se basan en el reconocimiento de la aversión que genera en los consumidores el sentirse atacados o manipulados por la publicidad en todo momento. El tercero se centra en tratar de impulsar el producto que se quiere vender a través de hacerlo destacar por medio de diferentes factores de la competencia. El cuarto es una mezcla de los anteriores, en donde se busca destacar una sensación o reacción pero sin ser evidente, tomando como blanco el subconsciente (Krishna y Schwarz, 2014).

En cualquier caso, estas y otras estrategias obedecen a la adaptación de la manera de comunicar a comportamiento de los clientes en los diferentes segmentos del mercado; en este sentido, es posible entonces ligar al marketing diferentes reglas o normas con base en el comportamiento humano, como lo exponen Ries y Trout (1999) al hablar de sus 22 leyes inmutables del marketing, asegurando que así como "usted puede construir un avión precioso; pero no despegará del suelo a menos que cumpla las leyes de la física y especialmente la de la gravedad" (Ries y Trout, 1999), se puede diseñar un plan de marketing, pero si no cumple con unas leyes fundamentales basadas en la percepción de las personas que lleva a tener éxito a la hora de posicionar una marca, no sirve de nada.

En este mismo sentido, Ariely (2008), en su libro Las trampas del deseo, describe la economía actual como una "economía conductual" y asegura que los seres humanos "no solo somos irracionales, sino previsiblemente irracionales; es decir, que nuestra irracionalidad se produce siempre del mismo modo una y otra vez" (Ariely, 2008). Esta condición se traduce en diferentes efectos que pueden ser activados haciendo uso de estrategias de marketing, y que funcionan en la mayoría de los casos para que las personas tomen la decisión deseada y diseñada por parte de quien las implementa. Estos modos de actuar se presentan en todos los animales. Se denominan modelos de acción fija y tienen como principal característica que "los comportamientos que los componen se desarrollan prácticamente de la misma forma y en idéntico orden siempre que aparecen. Es como si los modelos estuviesen grabados en una cinta dentro de los animales" (Cialdini, 1990). Para el caso específico, se trata de una cinta que puede ser reproducida en los seres humanos.

Con el objetivo de mostrar las influencias de los diferentes efectos que disparan conductas repetitivas en las personas a la hora de comportarse en un mercado, el grupo de investigación GEIO se dispone a crear una metodología bajo el marco del aprendizaje constructivista, que permita a los participantes reconocer un entorno en el que están presentes diferentes estrategias de marketing.

El principal investigador que dio vida a la teoría del constructivismo fue Piaget (1972, citado en Prince y Felder, 2006) con el constructivismo cognitivo, quien indica que las reacciones de un individuo a las experiencias conducen al aprendizaje (o no lo hacen). Y desde el constructivismo social, el lenguaje y las interacciones con otros juegan un papel primordial en la construcción del significado a partir de la experiencia, el significado es coconstruido (Prince y Felder, 2006).

Se considera entonces al constructivismo como la metodología adecuada para la actividad a plantear debido a lo mencionado anteriormente, y a que quien construye el conocimiento dentro de este paradigma es, según González-Tejero y Pons (2011):

[...] El sujeto activo que interactúa con el entorno y que, aunque no se encuentra completamente constreñido por las características del medio o por sus determinantes biológicos, va modificando sus conocimientos de acuerdo con ese conjunto de restricciones internas y externas. (p. 4)

La aplicación de la metodología constructivista en el grupo de investigación, también para el caso de la presente propuesta, se hace a través del juego serio, serious game, o juego formativo, denominado en GEIO como "lúdica", que tiene elementos constructivistas claros como la participación activa y central del estudiante, la interacción y trabajo grupal (entre pares y con los docentes) y el contexto estructurado, experiencial y auténtico que simula situaciones reales. Así, se permite la integración de conocimientos de diversas áreas y la experimentación de múltiples alternativas dentro del juego. Ariffin et al. (2014) afirman que el propósito de un juego serio es facilitar los procesos de enseñanzaaprendizaje en las organizaciones, y cumple sus objetivos mediante la inclusión de reglas, restricciones y actividades que replican de cerca las limitaciones de las tareas del mundo real. 
En la Tabla 1 se presentan los diferentes conceptos necesarios para entender la metodología a proponer, extraídos del libro Las trampas del deseo (Ariely, 2008) y explicados finalmente por los autores del presente artículo.

Tabla 1

Definiciones abordadas en la metodología

\begin{tabular}{|c|c|}
\hline Término & Definición \\
\hline Economía conductual & $\begin{array}{l}\text { Se basa en elementos tanto de la psicología como de la economía, partiendo de la idea de que las personas no } \\
\text { son completamente racionales. Además, no solo son irracionales, sino también previsiblemente irracionales; } \\
\text { es decir, que nuestra irracionalidad se produce siempre del mismo modo una y otra vez. }\end{array}$ \\
\hline Efecto señuelo & $\begin{array}{l}\text { Estrategia utilizada para cambiar la preferencia que pueden tener las personas hacia dos opciones al agregar } \\
\text { una tercera opción (señuelo) con características inferiores a una de las dos opciones iniciales, con el fin de que } \\
\text { estas se inclinen hacia la opción que resulta superior al señuelo. }\end{array}$ \\
\hline Efecto anclaje & $\begin{array}{l}\text { Valor que se acostumbra a pagar por un bien o un servicio. Se puede fijar con una primera compra, intención } \\
\text { de compra, publicidad u opinión de otra persona; así entonces, será la base para juzgar lo asequible o costoso } \\
\text { del mismo producto o servicio, al igual que productos o servicios similares, en ocasiones futuras. }\end{array}$ \\
\hline Coherencia arbitraria & $\begin{array}{l}\text { Es el condicionamiento de los precios futuros o que no se conocen, debido a la formación de un precio ancla. } \\
\text { Al saber cuánto se pagaría por un producto o servicio, se cree saber cuánto pagar por cinco productos, o por } \\
\text { un producto semejante ante una necesidad de compra futura. }\end{array}$ \\
\hline Gregarismo o efecto arrastre & $\begin{array}{l}\text { Es el sesgo en una decisión de compra producido por el conocimiento o la suposición, de la experiencia de otras } \\
\text { personas con este producto o servicio. La gente tiende a apoyar la causa que es aparentemente ganadora. }\end{array}$ \\
\hline Efecto coste cero & $\begin{array}{l}\text { Ocurre cuando la presencia de un bien o servicio gratis nos inclina desmedidamente a adquirirlo, tomando a } \\
\text { menudo decisiones erróneas o innecesarias. Se atribuye al intrínseco miedo que poseen los seres humanos a } \\
\text { perder, porque "cuando compras algo que no cuesta nada, no pierdes nada". }\end{array}$ \\
\hline Marketing emocional & $\begin{array}{l}\text { Un marketing que propone experiencias capaces de generar emociones que le lleven a interactuar con la } \\
\text { marca, dentro de un escenario que apela a sus motivaciones y deseos más profundos. }\end{array}$ \\
\hline $\begin{array}{l}\text { El efecto de la expectativa } \\
\text { o efecto Pigmalión }\end{array}$ & $\begin{array}{l}\text { Es el proceso por el cual las creencias y expectativas de una persona afectan la conducta y percepción, } \\
\text { tanto propia como colectiva; esto genera un sesgo y termina por modificar nuestras decisiones al generar } \\
\text { estereotipos y prejuicios. }\end{array}$ \\
\hline Material P. O. P. & $\begin{array}{l}\text { Es el material utilizado para promocionar productos y/o servicios en el punto de venta, haciéndolo más notorio } \\
\text { y resaltando características de este. }\end{array}$ \\
\hline Cenefa & $\begin{array}{l}\text { Elemento decorativo largo y estrecho que delimita el área de exhibición del producto del de la competencia, } \\
\text { destaca su presencia e informa sobre el precio. }\end{array}$ \\
\hline
\end{tabular}

Fuente: Autores con base en Ariely (2008).

\section{Metodología}

El primer paso en el diseño de la lúdica fue la documentación. Para esto se revisaron diversos textos y libros. La guía principal fue el libro Las trampas del deseo, de Ariely (2008). De este documento se obtuvieron las principales estrategias de marketing que se utilizan en el mercado y su respectiva definición. Se hizo necesario realizar un resumen de todos los conceptos, para poder asimilar mejor la información, además de llevarlos a lo más simple para idear situaciones en las cuales estos se pueden ver aplicados. Luego se buscó la manera de adaptar los conceptos a una metodología diseñada bajo el constructivismo, de acuerdo con lo expuesto en el marco referencial del presente documento.

En este caso, el escenario más apto para la simulación es un supermercado, debido a que no solo permite observar la teoría aplicada sino también interactuar con ella, de tal forma que los participantes puedan vivenciarlo de una manera más dinámica. Para que lo anterior sea posible, es necesario asignar correctamente los productos a cada experimento de acuerdo con las características que cada uno presenta y que permiten mostrar mejor los efectos.

\section{Tipo de estudio}

Este estudio es de tipo descriptivo, no experimental y de corte transversal, dado que se detalla el proceso de diseño de un ejercicio gamificado en un momento determinado, a partir de la observación y la investigación en fuentes secundarias. De la misma manera, mediante la investigación anteriormente mencionada, en el marco teórico, se llegó a una hipótesis y una metodología que teóricamente pueden solucionar las cuestiones planteadas, se tuvo especial énfasis en esta parte, y a pesar de no realizar pruebas para comprobarla, se determinaron los parámetros con los que se evaluará el cumplimiento de los objetivos, en caso de hacerse un estudio experimental posterior (Salinas, 2012).

\section{Resultados}

\section{Estructura y plan de la lúdica}

Los participantes estarán organizados en grupos de tres personas, tendrán una lista de necesidades para saber qué deben comprar, y después se les entregara dinero didáctico (GEIO gold) para comprar productos teniendo como base una lista de necesidades que corresponde 
Angie Paola Agudelo Loaiza, Catalina Londoño Velásquez, Ginette Peláez Rodríguez, Juan Pablo Méndez Ríos, Julián Andrés Gutiérrez Castaño Metodología para identificar las estrategias de mercadeo que afectan en el momento de compra: TRAPPOLA

a las compras mensuales de su equipo. Dentro del supermercado también irán sucediendo una serie de eventos que buscarán modificar el comportamiento de los participantes.

La lúdica se correrá por intervalos de 4 minutos por persona, donde cada una deberá satisfacer 3 necesidades. Al final de cada ronda se dispondrá de un corto tiempo para que cada integrante socialice sus decisiones de compra.

Para el desarrollo de esta lúdica, debe haber mínimo dos monitores que cumplan dos funciones. La primera será la de cajero, que deberá registrar los productos y posteriormente hacer la recolección y respectivo análisis de los datos. El observador, por su parte, servirá como apoyo para todos los grupos que se encuentren realizando la actividad; adicionalmente supervisará el desarrollo de esta para prevenir y evitar distorsión en los resultados debido al incumplimiento de las reglas de la lúdica.

El esquema de realimentación se divide en dos partes. La primera corresponde a la socialización por grupo donde hablarán acerca del presupuesto y el porcentaje usado en cada grupo de productos, como aseo o galguería y deberán explicar el porqué. En la segunda parte, se les comunicará a los grupos la finalidad real de la lúdica, además de explicar las diferentes "trampas" de marketing en las que acaban de caer y se les preguntará cómo fue su experiencia con base en las siguientes preguntas:

- ¿Qué tuviste en cuenta a la hora de elegir productos?

- Menciona dos trampas en las que caíste

- ¿En qué trampas no caíste y por qué?

- ¿Puedes pensar en escenarios en tu vida donde hayas vivenciado estos efectos?

- ¿Cómo podrías evitar estas trampas?

\section{Criterios de evaluación}

En caso de querer evaluar los resultados de la lúdica se requiere tener en cuenta distintos criterios como:

- Qué tanto de la información pudieron retener las personas.

- Si serían capaces de evitar caer en las mismas trampas.

- Los participantes se interesaron por el tema como para querer aprender más acerca de este.

Para evaluar todos los criterios de manera general están las preguntas que se realizarán al final de la actividad, donde se podrá ver qué conceptos asimilaron, si son capaces de identificar las trampas y si pueden proponer soluciones propias para evitarlas. Además, se evalúa también qué tanto interés tiene por seguir aprendiendo acerca del tema. Sin embargo, para este método de evaluación solo nos permite conocer de manera general si se cumplieron los objetivos. En caso de que se desee hacer un estudio experimental acerca de la metodología para comprobar su efectividad, estos criterios y las preguntas serían la base sobre la cual crear las estrategias de toma de datos.

\section{Materiales de la lúdica}

A continuación, se listan los elementos necesarios para el desarrollo de la lúdica y los respectivos materiales empleados en su construcción:

Estanterías: El material usado en las estanterías es el cartón piedra, exactamente dos láminas de 70 cm* $100 \mathrm{~cm}$ para cada estantería. En total son 8 láminas, ya que se usarán 4 estanterías. En la Figura 1 se muestra el diseño digital de las estanterías.

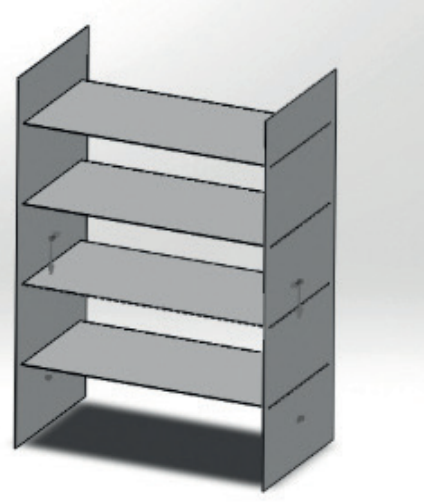

Figura 1. Diseño de estantería hecho en Solidworks. Fuente: Autores.

Tarjetas: Cada producto tendrá una cantidad determinada de tarjetas de presentación con su correspondiente código para hacer más fácil el proceso de "llevar" el producto hacia la caja registradora. Estas tarjetas serán plastificadas. El diseño digital de las tarjetas de presentación se muestra en la Figura 2.

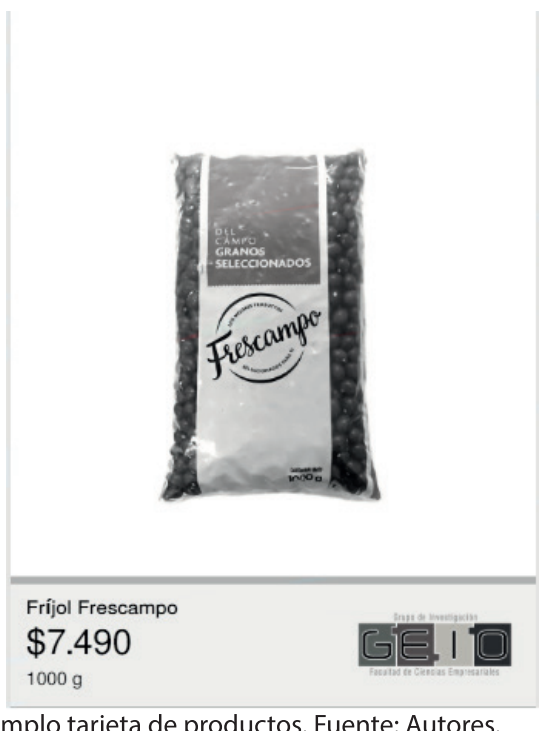

Figura 2. Ejemplo tarjeta de productos. Fuente: Autores. 
Material P. O. P.: Como en todo supermercado, algunos productos contarán con un material P. O. P. que será asignado dependiendo del efecto que se quiera demostrar. Para esta lúdica los dos tipos de materia P. O. P. que se van a utilizar serán cenefas y rompe pasos. Estos serán realizados mediante impresiones en cartulina que posteriormente serán pegadas a cartón para darles firmeza. En la Figura 3 se presenta uno de los diseños de rompe pasos.

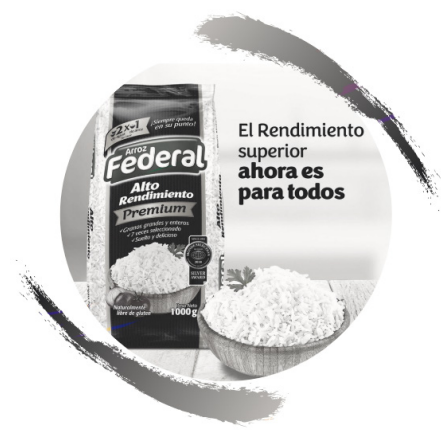

Figura 3. Diseño rompe pasos. Fuente: autores.

\section{Discusión}

Durante todo el proceso de investigación se detectó un problema relacionado con el tema del marketing, a partir del cual se logró concluir que existe una gran falta de conocimiento acerca de las estrategias de marketing por parte del consumidor promedio, tal como lo sugieren autores como Ariely (2008).

Después de un segundo proceso de investigación, se diseñó una metodología basada en situaciones de la cotidianidad que permitiera compartir todos estos nuevos conceptos, y que además capture la atención de las personas, para que puedan asimilar la información más fácilmente. Esto, en teoría, solucionaría el problema de estudio; sin embargo, es necesario realizar pruebas para poder afirmarlo.

Además, esta metodología se resumió y se especificaron todos los pasos necesarios para la implementación de esta, para que más adelante personas que no hayan sido parte del diseño puedan igualmente implementarla y así impactar a muchas más personas.

Llevar este conocimiento a las personas y permitirles que lo vivan personalmente logra, en teoría, generar un interés genuino hacia el tema y despertar curiosidad, para que se siga aprendiendo acerca de este.

\section{Conclusiones}

Se concluye que es necesario desarrollar lúdicas enfocadas en el área de marketing, pues existen muchos conceptos importantes e interesantes de los cuales aún no se han creado metodologías para su enseñanza y familiarización.

Gracias a las distintas fuentes bibliográficas consultadas, se estableció que el comportamiento humano es predecible, y esto permite que el marketing cumpla su objetivo en la mayoría de los casos (Akdoğan y Altuntaş, 2015).

Cabe mencionar que el estudio es un punto de partida, en donde se presentan las bases teóricas, se diferencia un problema, y se desarrolla una solución plausible, además de dejar unos criterios para en un futuro poder ampliar el estudio con una parte experimental. En esta se implementará la metodología varias veces, y de esta forma se recolectarán datos para obtener conclusiones respaldadas por estos. Actualmente se analiza la mejor manera de recolectar datos, y se espera empezar con la parte experimental cuanto antes.

Se espera que la investigación impacte de manera positiva en la comunidad de estudiantes de la universidad en la cual se desarrolló la metodología, además de todas las personas a las que se pueda llegar.

La importancia de esta investigación recae en demostrar la idea de que, con técnicas psicológicas simples pero bien fundamentadas, se puede predecir y guiar el comportamiento de las personas en un ambiente controlado y cotidiano. El que las personas vivan personalmente esta experiencia es la manera más fácil y entretenida de transmitir los conocimientos acerca del tema y generar un interés.

La metodología creada sería la primera del área de marketing dentro del grupo de investigación GEIO de la facultad de ciencias empresariales de la universidad donde se desarrolló.

\section{Agradecimientos}

Se agradece al grupo de investigación GEIO de la Universidad Tecnológica de Pereira por la asistencia y ayuda durante la realización del estudio, y en general a todos los integrantes del área de marketing del grupo de investigación GEIO, que han contribuido de alguna forma para llevar a cabo el estudio.

\section{Referencias}

Akdoğan, M. Ş. y Altuntaş, B. (2015). Covert Marketing Strategy and Techniques. Procedia-Social and Behavioral Sciences, 207, 135-148.

American Marketing Association. (2017). Definitions of Marketing. https://www.ama.org/the-definition-ofmarketing-what-is-marketing/ 
Angie Paola Agudelo Loaiza, Catalina Londoño Velásquez, Ginette Peláez Rodríguez, Juan Pablo Méndez Ríos, Julián Andrés Gutiérrez Castaño Metodología para identificar las estrategias de mercadeo que afectan en el momento de compra:TRAPPOLA

Anderson, C. (2007). La economía Long Tail: De los mercados de masas al triunfo de lo minoritario. Editores Urano S.A.

Ariely, D. (2008). Las trampas del deseo. Ariel.

Ariffin, M. M., Oxley, A. y Sulaiman, S. (2014). Evaluating Game-Based Learning Effectiveness in Higher Education. Procedia-Social and Behavioral Sciences, 123, 20-27. https://doi.org/10.1016/j. sbspro.2014.01.1393

Cialdini, R. (1990). Influencia ciencia y práctica. Cuáles son los factores determinantes para que una persona

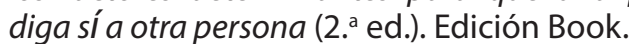

Godin, S. (2002). La vaca purpura: Diferénciate para transformar tu negocio. Gestión 2020.

González-Tejero, J. M. y Pons, R. M. (2011). El constructivismo hoy: Enfoques constructivistas en educación. Revista Electrónica de Investigación Educativa, 13(1), 1-27.

Kotler, P. y Keller, K. (2006). Dirección de marketing (12. ${ }^{a}$ ed.). Pearson educación. https://frrq.cvg.utn. edu.ar/pluginfile.php/14585/mod_resource/ content/1/librodireccion-de-marketing\%28kotlerkeller_2006\%29.pdf

Krishna, A. (2012). An Integrative Review of Sensory Marketing: Engaging the Senses to Affect Perception, Judgment and Behavior. Journal of Consumer Psychology, 22(3), 332-351.

Krishna, A. y Schwarz, N. (2014). Sensory Marketing, Embodiment, and Grounded Cognition: A Review and Introduction. Journal of Consumer Psychology, 24(2), 159-168. https://doi.org/10.1016/j. jcps.2013.12.006

Piaget, J. (1972). The Psychology ofthe Child. Basic Books.

Prince, M. J. y Felder, R. M. (2006). Inductive Teaching and Learning Methods: Definitions, Comparisons, and Research Bases. Journal of Engineering Education, 95(2), 123-138. https://doi. org/10.1002/j.2168-9830.2006.tb00884.x

Ries, A. y Trout, J. (1999). 22 leyes inmutables del marketing. McGraw Hill.

Roy, A. y Chattopadhyay, S. P. (2010). Stealth Marketing as a Strategy. Business Horizons, 53(1), 69-79. https:// doi.org/10.1016/j.bushor.2009.09.004

Salinas, P. (2012). Metodología de la investigación científica. Universidad de Los Andes. https://n9.cl/7nk3 